\title{
Finite element modeling of ejection cracks in powder metallurgy die compaction processes: a case study
}

\author{
J.A. Hernández ${ }^{\mathrm{a}, \mathrm{c}, *}$, J. Oliver ${ }^{\mathrm{b}}$, J.C. Cante ${ }^{\mathrm{c}}$, R. Weyler ${ }^{\mathrm{c}}$ \\ ${ }^{a}$ Centre Internacional de Mètodes Numèrics en Enginyeria (CIMNE), U.P.C, Edificio C1, Campus Norte, \\ Jordi Girona 1-3, Barcelona 08034, Spain \\ ${ }^{b}$ E.T.S. d'Enginyers de Camins, Canals i Ports, Technical University of Catalonia, Edificio C1, Campus \\ Norte, Jordi Girona 1-3, Barcelona 08034, Spain \\ ${ }^{c}$ E.T.S. d'Enginyeries Industrial i Aeronàutica de Terrassa, Technical University of Catalonia, C/ Colom, \\ 11, Terrassa 08222, Spain
}

\begin{abstract}
To date, the modeling of crack formation during the ejection stage in Powder Metallurgy $(\mathrm{P} / \mathrm{M})$ die compaction processes has fallen outside the scope of conventional finite elements studies on this process. In this paper, we attempt to make a first exploratory step in this regard by presenting a carefully detailed case study that exemplifies how crack simulations can be harnessed to solve real $\mathrm{P} / \mathrm{M}$ manufacturing problems. The part subjected to study is a multilevel adapter whose design-to-production process proved problematic to the manufacturer, to the point that simplifications in the geometry of the part were to be made to avoid the appearance of cracks during ejection. The goal here is to, through finite element analysis of several, alternative ejection schemes, clarify the reasons behind the difficulties in ejecting free-crack compacts with the original geometry; understand the connection of such difficulties with the geometry modification introduced in the revised design; and, on the basis of the insight gained from the computed results, make recommendations on the prevention of similar problems in other situations.
\end{abstract}

Key words: POWDER METALLURGY; FINITE ELEMENT MODELING; EJECTION; CRACKING

\footnotetext{
${ }^{*}$ Corresponding author

Email address: jhortega@cimne.upc.edu (J.A. Hernández)
} 


\section{Introduction}

Two relatively recent and fairly comprehensive reviews on finite element modeling of Powder Metallurgy (P/M) die compaction processes $[1,2]$ have concluded that, while the accuracy that has been achieved in predicting final densities and punch forces can be deemed satisfactory from a practical point of view, there are still several predictive and functional deficiencies that limit the extensive take-up of finite element simulation by the $\mathrm{P} / \mathrm{M}$ industry. One of the chief weaknesses pinpointed in these reviews is the inability of available computational models to anticipate the structural integrity of the green compact, that is, the inability to predict the formation of cracks.

Cracking in green compacts is one the major quality problems experienced by the $\mathrm{P} / \mathrm{M}$ industry; it is perhaps the bête noire of ferrous components manufactures. Cracks formed during pressing operation — due to insufficient interparticle bonding, mass slippage, etc. — can be prevented by devising pressing schedules that ensures a nearly-uniform density distribution, or at least avoid sharp density gradients; the density prediction ability of available finite element models can greatly assist the $\mathrm{P} / \mathrm{M}$ designer in this task, as shown, for instance, in Ref. [3]. The first serious attempt to numerically model cracking in $\mathrm{P} / \mathrm{M}$ compaction, made by [4], and also most of later proposals (see for instance [5]), have focused exclusively on the simulation of these "pressing" cracks.

Very frequently, however, cracks in green compacts are not the manifestation of an inappropriate consolidation process, but rather the result of improper pressure release and/or ejection (from the die cavity) stages; the poor tensile properties of green parts render their structural integrity exquisitely vulnerable to tensile/shear stresses provoked by improper punch deflections or excessive elastic spring-back. In contrast to the modeling of "pressing" cracks, the numerical study of cracking during pressure release and ejection in $\mathrm{P} / \mathrm{M}$ die compaction processes has been scarcely considered in the $\mathrm{P} / \mathrm{M}$ literature. Earlier attempts to predict post-pressing failure focused on merely analyzing the evolution of stresses throughout the part so as to detect excursions above a given stress threshold, yet without giving any hint on crack propagation features such as length, orientation, severity, etc. [6] and [7], 
for instance, use simple, experimentally obtained fracture criteria based on the maximum principal stress. To actually model crack propagation, it is necessary to enrich the constitutive model with some means to capture the deterioration of strength experienced by the material after the attainment of the corresponding yield or fracture threshold; namely, a strain-stress softening law. The work by [8] stands as a landmark in this regard, since it provides the first, to the authors' knowledge, experimental measurements of fracture energy - an indispensable ingredient for the calibration of the aforementioned softening law of consolidated iron-based powder. In [9], a fictitious crack model featuring a stress versus crack-width relation dependent on the fracture energy values obtained in [8] is proposed and successfully applied to reproduce cracking in a diametral compression test; however, no critical assessment of its performance in a real manufacturing scenario is carried out, n3or is it addressed the theoretical formalisms behind the inescapable coupling between such highly non-linear phenomena as densification and cracking — the higher the density attained locally, the higher the strength and the toughness, and hence the resistance to the propagation of cracks. The present authors have elaborated elsewhere $[10,11]$ a constitutive framework that do permit to describe these two conspicuously distinct phenomena in a thermodynamically sound, unified manner. A radically distinct approach to crack prediction in $\mathrm{P} / \mathrm{M}$ compacts, on the other hand, is adopted in the work of Rolland et al. [12]. They address the problem in a probabilistic fashion: rather than treating the occurrence of cracks as a "deterministic" event, Rolland et al. account for the inherent randomness and uncertainty of material parameters and tooling kinematics and use a failure risk model that enables one to identify and delimit areas of the part where cracks are likely to develop.

Despite these recent efforts to improve the cracking-predictive ability of die compaction models, the topic remains still within the research arena, and crack simulation technology is not yet being exploited by the $\mathrm{P} / \mathrm{M}$ industry to tackle problems linked with cracking during ejection in die compaction processes. It is precisely the purpose of the present paper to attempt to make a first exploratory step in this regard by presenting a detailed case study that exemplifies how crack simulations can be used to address real $\mathrm{P} / \mathrm{M}$ manufacturing problems. The finite element program used for this purpose has at its core the abovementioned 
constitutive model developed by the authors in [10]. The part subjected to study is an axisymmetric, low-height, multilevel adapter (see figure 1) whose design-to-production process proved dramatically problematic, i.e. costly and time-consuming, to the manufacturer: although almost every reasonable and technically realizable alternative was tried, it was not possible to find a single ejection procedure leading to free-crack compacts. In order to obtain a reliable product, the manufacturer was finally compelled to abandon the fabrication of the part in its original form and agree with the client certain modifications - inconsequential to the functionality of the finished component - in the initial geometry. The result of these modifications is shown in figure 2: the abruptness of the cross-sectional change between the outermost levels is eliminated by a tapered surface. The goal of the present study is to, by interpreting the outcome of finite element simulations of various, alternative ejection schemes, and admittedly with the advantage of hindsight, throw some light on the possible reasons behind such difficulties; understand their connection with the geometry modification introduced in the revised design; and, on the basis of the insight gained from the computed results, make recommendations on the prevention of similar problems in other situations. This case study will serve also to provide some useful guidelines and advises on the modeling of pressure release and ejection in computed numerically controlled (CNC) advanced press machines, and on how computed results should be interpreted in order to arrive at accurate diagnosis and avoid misleading claims regarding the adequacy of a given ejection route.

\section{Preliminaries}

\subsection{Constitutive model}

The powder constitutive model used for the computations is basically an extension and refinement - to cover also the description of material failure - of the classical isotropic, elasto-plastic Drucker-Prager/Cap type model (see figure 3). Strain history is encapsulated into two internal variables: a hardening variable which is taken approximately as the relative inelastic density, and a softening variable $\xi^{s}$ associated with the accumulated (plastic) shear strain. When the stress state remains on the Drucker-Prager yield surface during interval of 

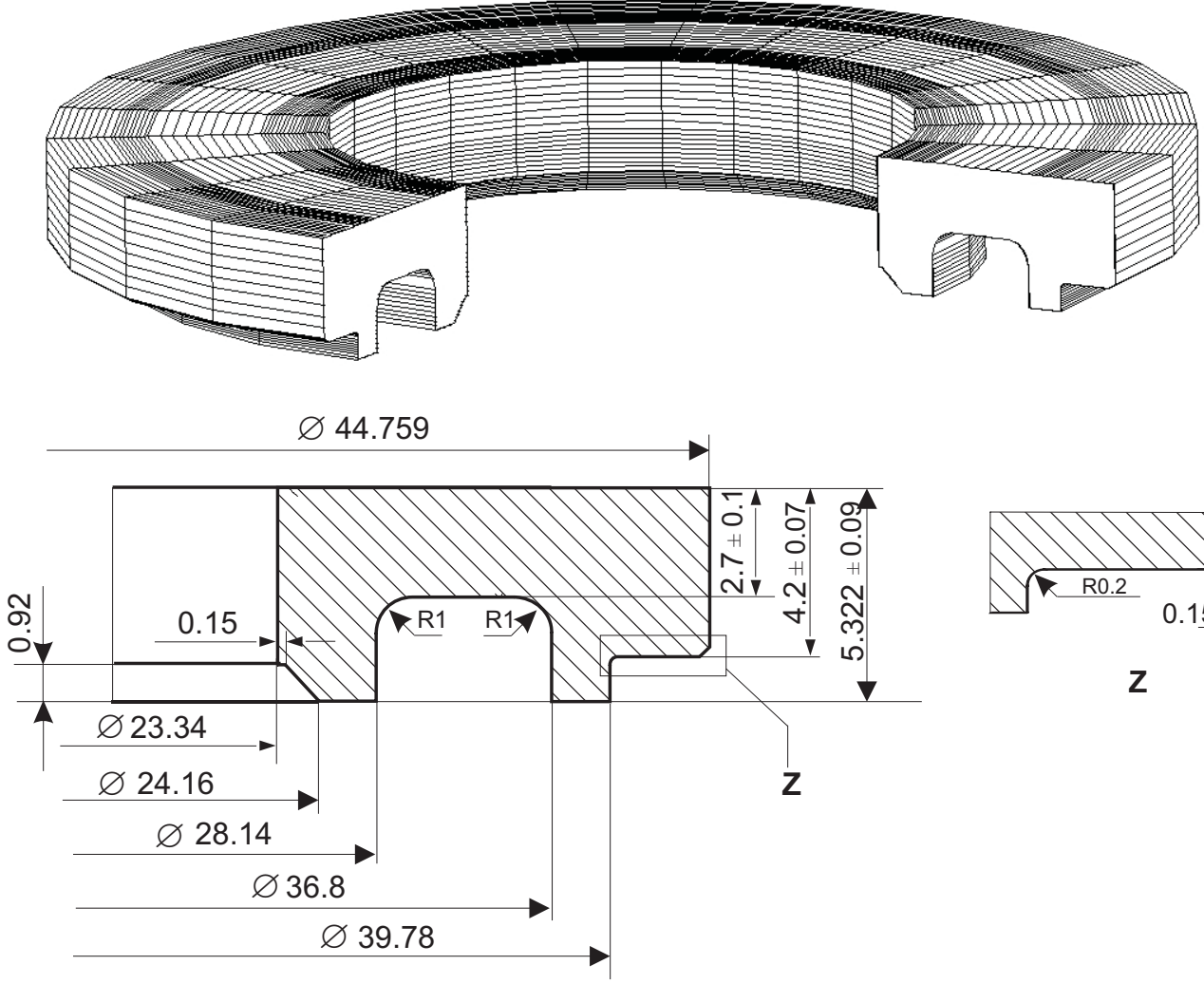

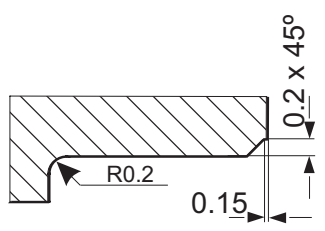

Z

Figure 1: Geometry of the part (dimensions in $\mathrm{mm}$ ). The axisymmetric geometry is revolved $270^{\circ}$ for ease of visualization.

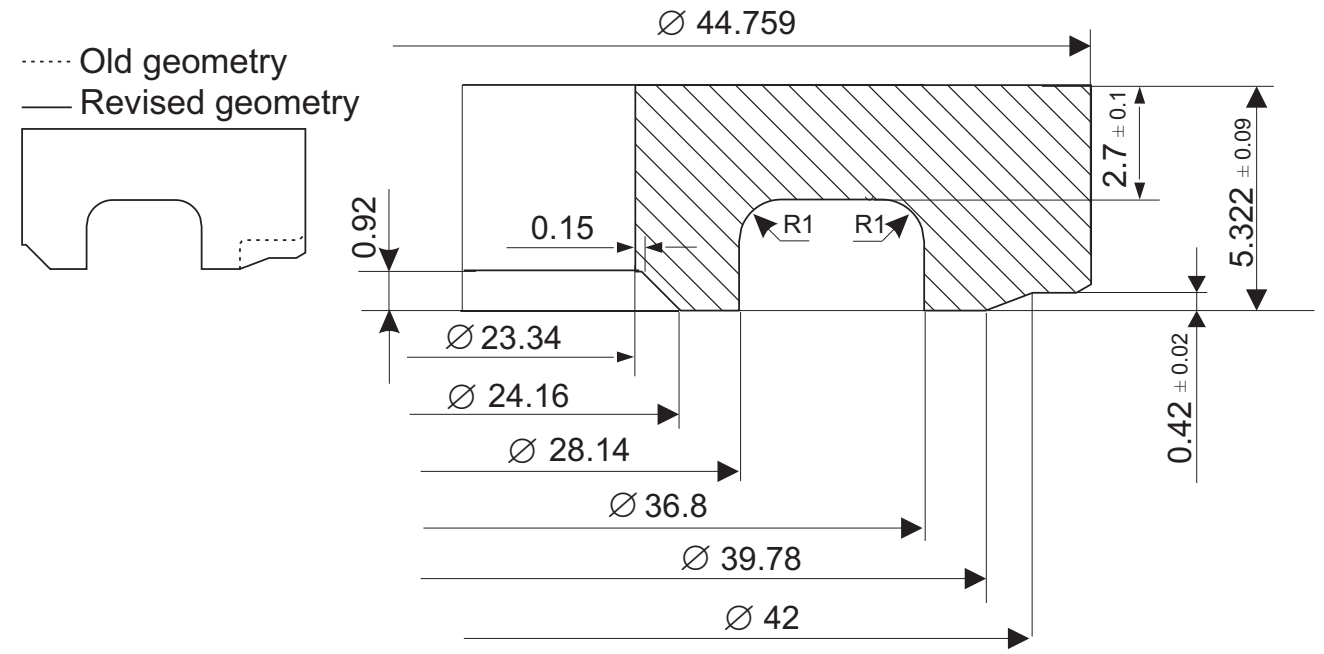

Figure 2: Revised geometry (dimensions in $\mathrm{mm}$ ). 


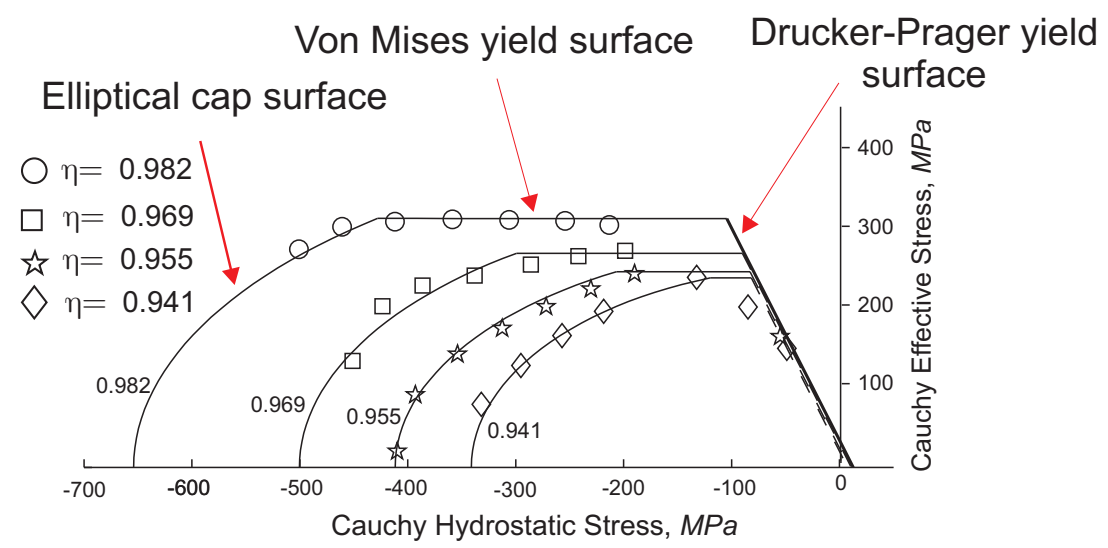

Figure 3: Experimental yield stress data (as a function of the relative density) from consolidated and overconsolidated compression tests on Distaloy AE powder specimens [13], together with isodensity contours constructed using a Drucker-Prager/Cap type criterion refined with a density-dependent Von Mises yield surface.

continued plastic loading, the internal softening variable $\xi^{S}$ increases its magnitude, causing, in turn, a decrease in the cohesion variable c - the intersection of the Drucker-Prager yield surface with the deviatoric axis. This cohesion variable can be regarded as a measure of the green strength of the compacting powder. The relationship between cohesion and internal softening variable is given by the following softening law:

$$
c\left(\xi^{h}, \xi^{s}\right)=c_{0}\left(\xi^{h}\right) \exp \left(\frac{H_{0} \xi^{S}}{c_{0}}\right)
$$

where $c_{0}$ stands for the initial cohesion and $H_{0}$ represents the softening parameter, whose calibration is made in terms of the fracture energy values (see introductory section) obtained in [8]. Crack locations will be identified in this work as localized ${ }^{1}$ areas - in computed contours of cohesion - where cohesion is ostensibly lower than in its surroundings.

For further details of the theoretical basis of the employed constitutive model, and of its numerical implementation in a finite element code, the reader is referred to [10] and [11], respectively.

\footnotetext{
${ }^{1}$ In the presence of softening behavior, plastic deformations tend to occur in localized regions accompanied by elastic unloading in the surrounding material (strain localization).
} 


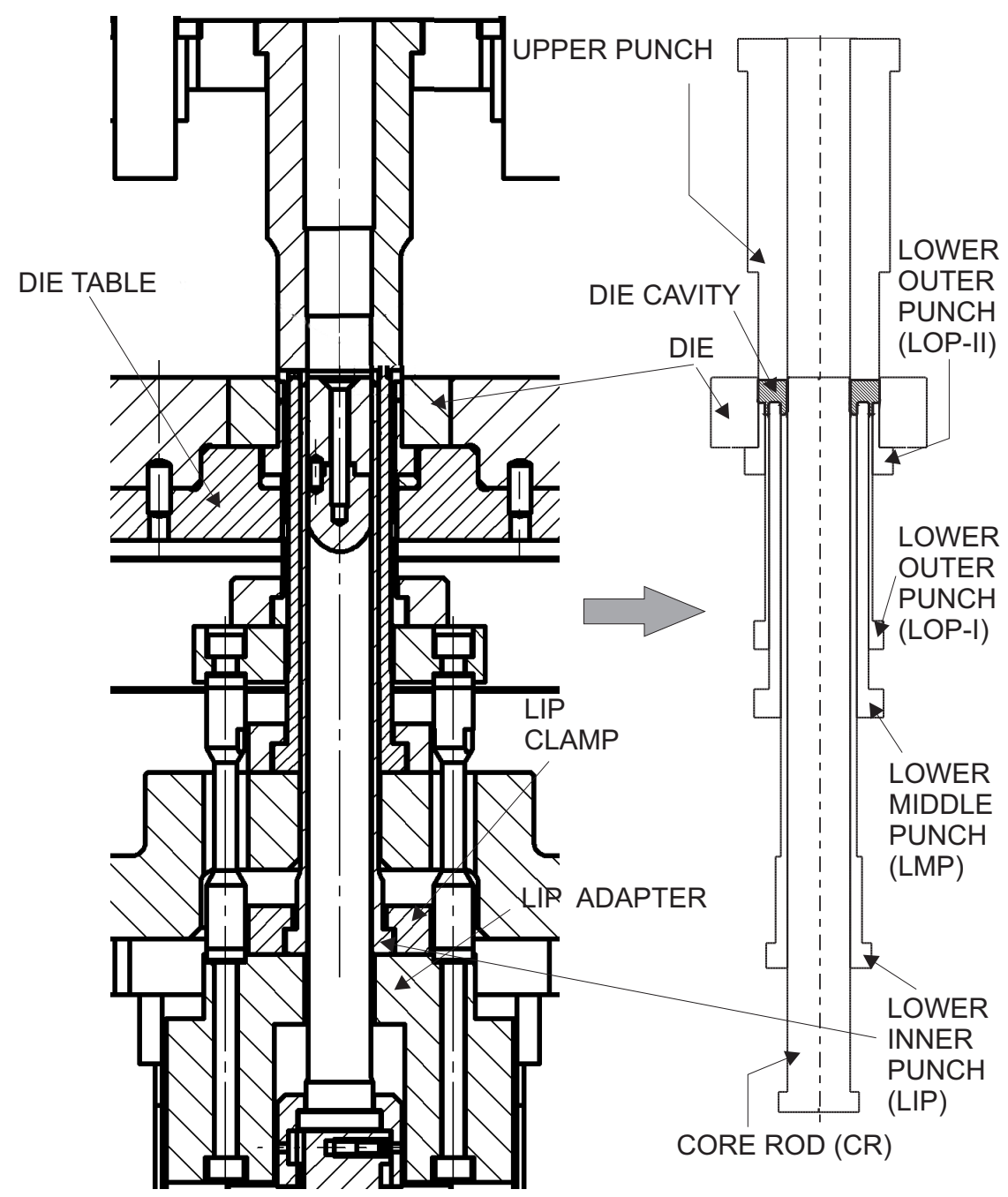

(a)

(b)

Figure 4: (a) Cross sectional view of the compacting press. (b) Geometric model of the tooling items included in the simulation.

\subsection{Description of the compacting press}

The schematic of the CNC, multi-platen press machine employed for compacting the part is shown in figure 4.a. In the accompanying drawing, figure 4.b, only those elements considered for modeling purposes are displayed: an upper punch (UP); four lower punches (LIP, LMP, LOP-I, LOP-II $)^{2}$; a die that controls the outer peripheral shape; and a core

\footnotetext{
${ }^{2}$ The acronyms LIP, LMP and LOP signify lower inner punch, lower middle punch and lower outer punch, respectively
} 
rod that controls the inner peripheral shape and size of the part. The upper punch is mechanically driven during pressing, but not during pressing pressure release and ejection; a hydraulically controlled mechanism, inserted between the upper punch and the upper ram allows independent movements during this stage. The lower punches labeled in figure 4.b as LIP, LMP and LOP-I, as well as the die and the core rod, are mounted on separate platens operated by hydraulic cylinders placed on the stationary member of the press; the lower outer punch LOP-II, by contrast, cannot move independently, but rather is attached to the die table in a stepped die fashion (or "shelf" die fashion, as termed by [14]).

\subsection{Powder and tooling properties}

The dimensions of the die cavity and the initial finite element mesh layout are displayed in figure 5. The powder employed in making the part is a Distaloy AE iron based powder with apparent density $\rho_{a p p}=3.25 \mathrm{~g} / \mathrm{cm}^{3}$. Material parameters can be obtained from the empirical adjustment presented in [10]. Fill density is assumed to be equal to the apparent density and uniform throughtout the die cavity. Friction between the powder mass and the faces of the tools-die walls and core rod is modeled via a friction Coulomb law, with coefficient $\mu=0.12$. The isotropic, linear, elastic behavior of the tooling is characterized by a Young's Modulus $E_{\text {tool }}=210 G P a$ and a Poisson's ratio $\nu_{\text {tool }}=0.3$.

\subsection{Pressing stage}

It need no emphasis that the success in predicting numerically the formation of cracks during the post-pressing stages (pressing release and ejection) relies considerably on the quality of the density and stress distributions computed at the end of the pressing stage. In a recent paper [11], the authors provide both an accurate description of the boundary and initial conditions for modeling the pressing of the concerned part and a critical assessment of finite element results - using the abovementioned constituve model — corresponding to this phase. Here, we limit ourselfs to show the computed density contours reported in such a paper (see figure 7). The portion with the lowest density is located above the lower inner punch; in this case, the numerically predicted value is $0.10 \mathrm{~g} / \mathrm{cm}^{3}$ below the experimental 


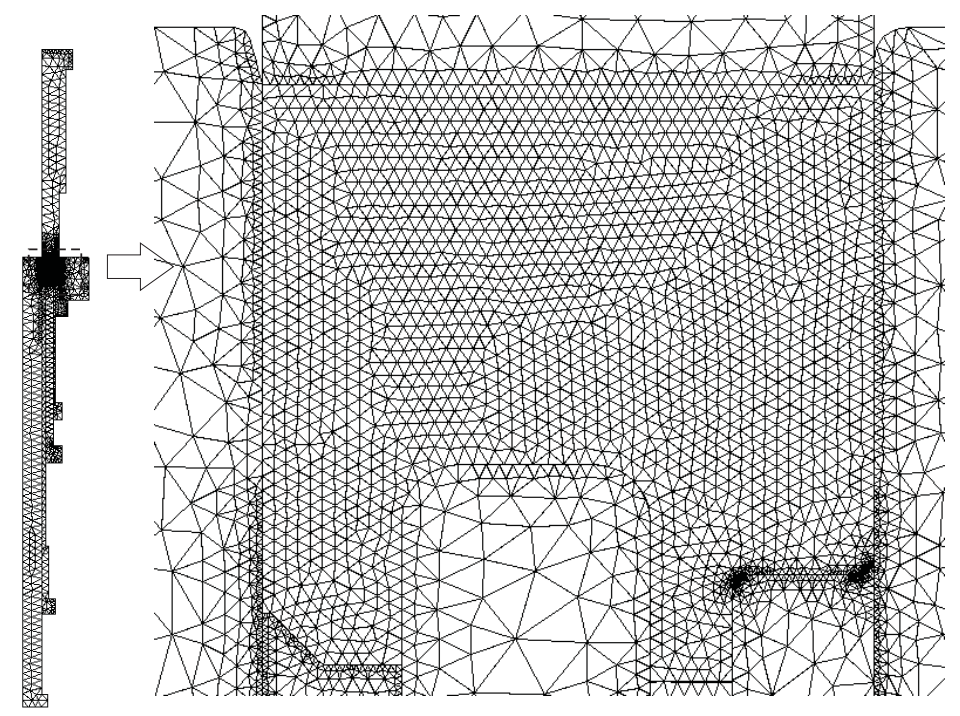

Figure 5: Initial mesh layout.

one, which is also the maximum discrepancy between experiment and predicted density values.

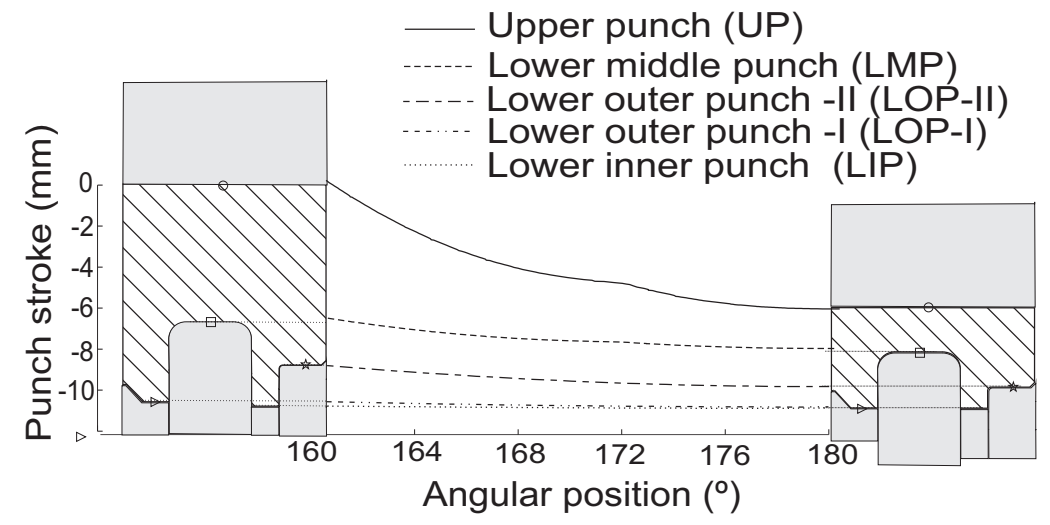

Figure 6: Tooling displacement as a function of angular position during pressing.

\section{Ejection of the original part}

The ejection stage covers approximately the portion of compaction cycle ranging from $180^{\circ}$ (end of pressing) to $265^{\circ}$ (where the part is picked up by the gripper), and can be further subdivided into three distinctly different phases; this division is illustrated schematically in figure 8 . The first phase corresponds to partial pressure release $\left(180^{\circ}\right.$ to $\left.190^{\circ}\right)$, where the 


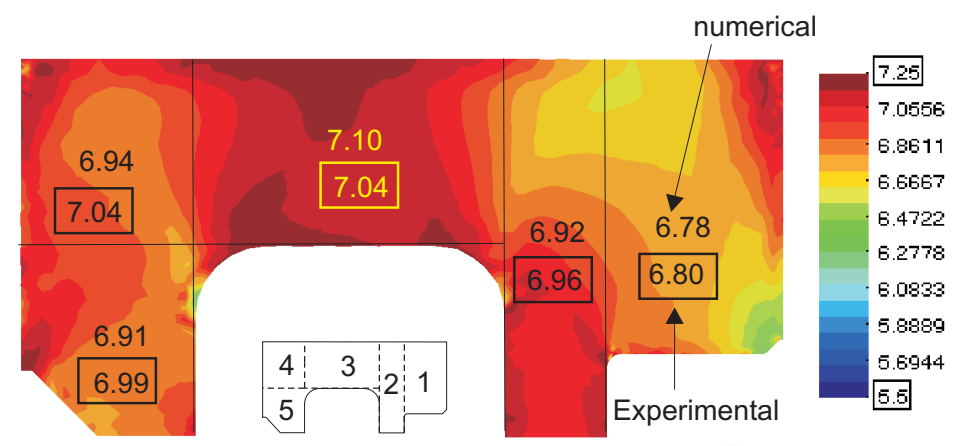

Figure 7: Contour plot of density computed at the end of the pressing stage.

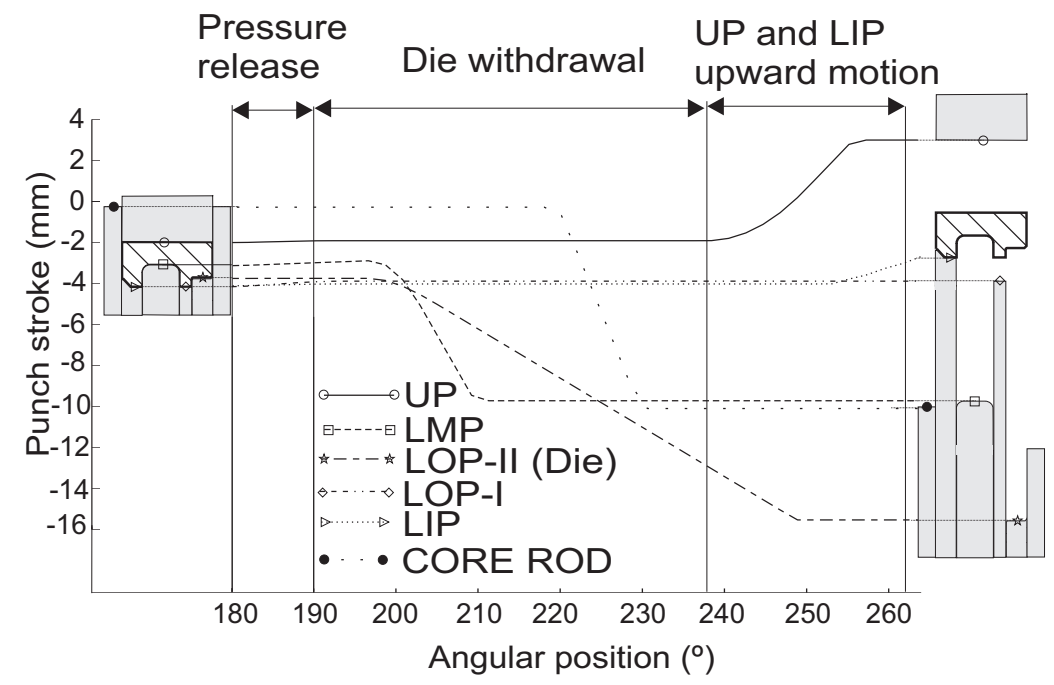

(b)

Figure 8: Typical tooling motion profile for the ejection stage.

upper punch moves slightly upward so as to reduce radial forces and facilitate the subsequent withdrawal of the die. In the second phase, from $190^{\circ}$ to approximately $240^{\circ}$, the die moves downward (withdrawal tooling system) while the two lower punches (LIP and LOP-I) forming the lowest faces remain stationary so as to support the part. In the third stage, ranging from $240^{\circ}$ to $265^{\circ}$, the upper punch moves up away from the compact and, simultaneously, the lower inner punch lifts the part slightly so that it can be removed by the gripper from the compaction zone. The finite element results shown in the sequel will be also presented according to this division of the process.

For illustrative purposes, a microscopic view of two of these observed cracks is shown in 
figure 9, although it should be pointed that the ensuing discussion is not intended to set up a systematic comparison between experimentally recorded crack images and computed cohesion contours; the retrospective character of the study makes somewhat elusive such rigorous verification of results. Rather, we limit ourselves to qualitatively examining, by simple comparison of cohesion contours, the adequacy of alternative ejection processes. Consider two alternative routes $A$ and $B$ for ejecting the part from the die cavity. If, for instance, the cohesion computed using the ejection route $A$ remains unaltered during the process, whereas the predicted cohesion contours using ejection schedule $B$ shows clear signs of cracking, we can, cautiously, claim that ejection schedule $A$ is, comparatively, and according to numerical predictions, more appropriate than ejection route $B$.

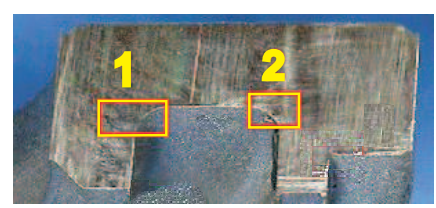

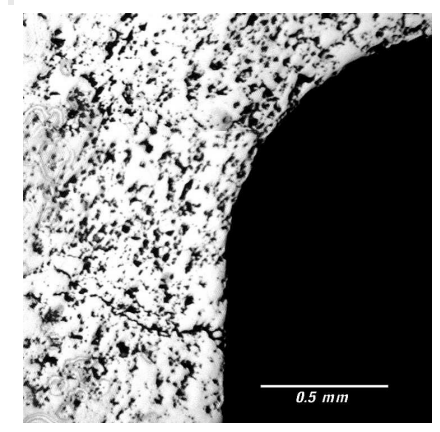

(a)

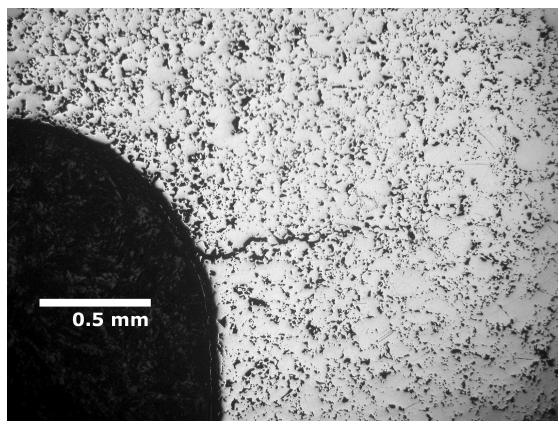

(b)

Figure 9: Cross-sectional view showing two cracks detected in the green compact. Microscopic image of: (a) Zone 1 (b) Zone 2

\subsection{Pressure release}

We mentioned earlier that the analyzed part was manufactured in a press machine in which, during the post-pressing operations, the upper punch is operated by a separate hydraulically driven system; this mechanism allows to apply a certain downward pressure during withdrawal of the die, the so-called hold down or counter-pressure. In the numerical simulation presented in the following, the upper punch is gradually lifted until the axial force 
on the upper punch is set to 10 Tons. The CNC press machines also offers the possibility

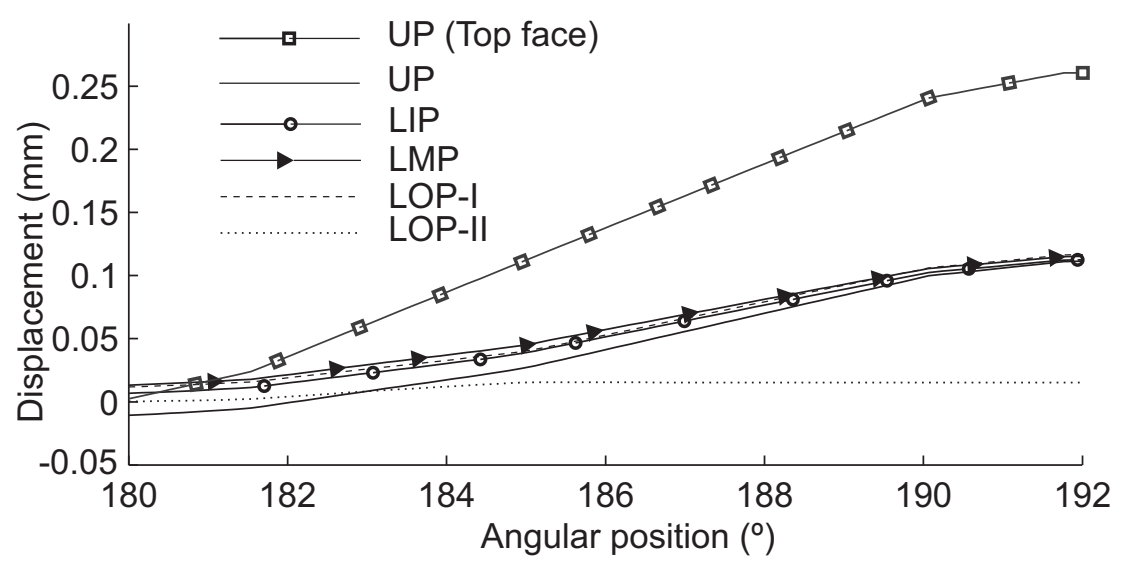

Figure 10: Displacement of the working ends of lower punches, as well as the displacement of the top face of the upper punch, as a function of the angular position (pressure release stage).

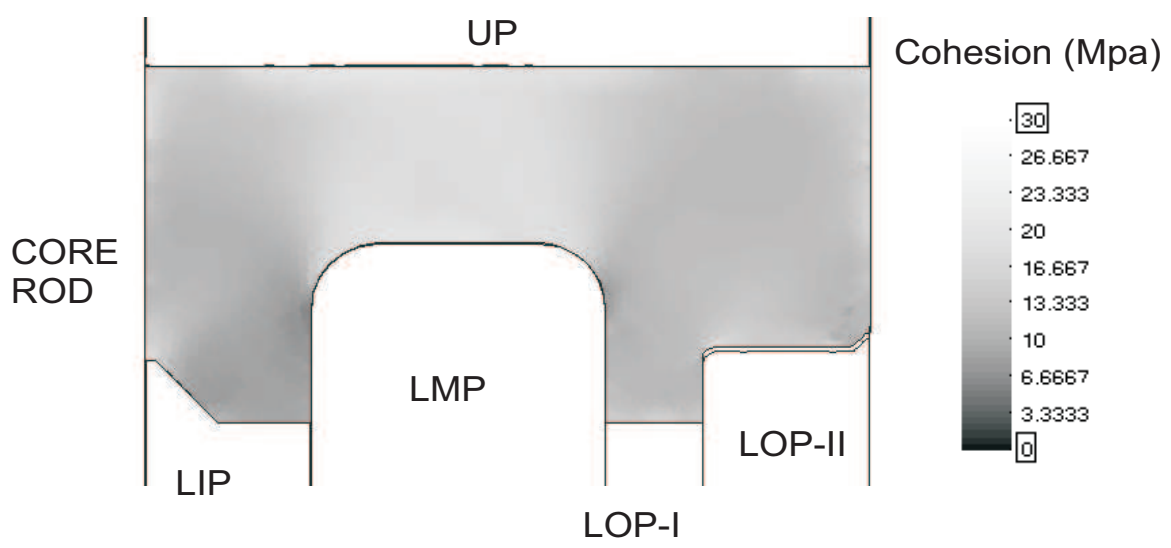

Figure 11: Contour plot of cohesion at the end of pressure release stage. Balanced deflection of lower punches case.

of accurately controlling the position of the lower rams. In order to compensate for the unbalanced punch deflections, the lower inner punch LIP, which is the largest one, descends $0.05 \mathrm{~mm}$, whereas the lower outer punch LOP-I moves $0.04 \mathrm{~mm}$ upward. The LMP and die platens are kept fixed. Figure 10 displays the computed vertical displacement, as a function of the angular position, of the punch working ends together with the displacement of the 
top face of the upper punch. According to the graph corresponding to the UP top face, the imposed reduction in axial force requires to move the upper punch $0.25 \mathrm{~mm}$ upward. The displacement curves corresponding to the working ends of the UP, LIP, and LMP-I meet at the end of the pressure release stage, a fact that indicates that the motions prescribed at the bottom of the LIP and LMP-I have proved efficient in compensating for the differences in elastic deflection between punches. The relative uniformity exhibited by the cohesion distribution computed at the end of pressure release stage, displayed in figure 11, confirms also this efficiency since no appreciable evidences of loss of cohesion are detected.

\subsection{Withdrawal of the die}

Using as starting point the results obtained at the end of the pressure release stage, we tackle now the analysis of the die withdrawal phase. Three different ejection procedures are numerically tested. In these three alternatives, the compact is held between the upper punch and the two lower punches forming the lowest faces of the compact (LIP and LOP-I), which act, thus, as supporting punches as the die is withdrawn.

\section{Option a: Held stationary the LMP and the core rod.}

We shall explore first the consequences of keeping fixed the lower middle punch and the core rod as the die is lowered. The tooling motion profile corresponding to this situation is depicted in figure 12. This diagram is accompanied by a sequence of three contour plots of cohesion computed at different times during the process. In the rightmost plot, which is shown also - in magnified form - in figure 13.a, a horizontal de-cohesion path stemming from the outer wall is observed. This de-cohesion pattern certainly exhibits a distinctly crack-like appearance. However, the factor or factors that provoke its development are not readily apparent. In order to identify these factors, and eventually clarify the mechanical origin, if any, of this localized loss of cohesion, we shall propose two alternative, physically plausible hypothesis.

The first hypothesis considers that the computed crack is due to the effects of elastic strain release in the radial direction. The observed mechanical degradation arises when the top face of the die is approximately flush with the top face of the lower middle punch, that 


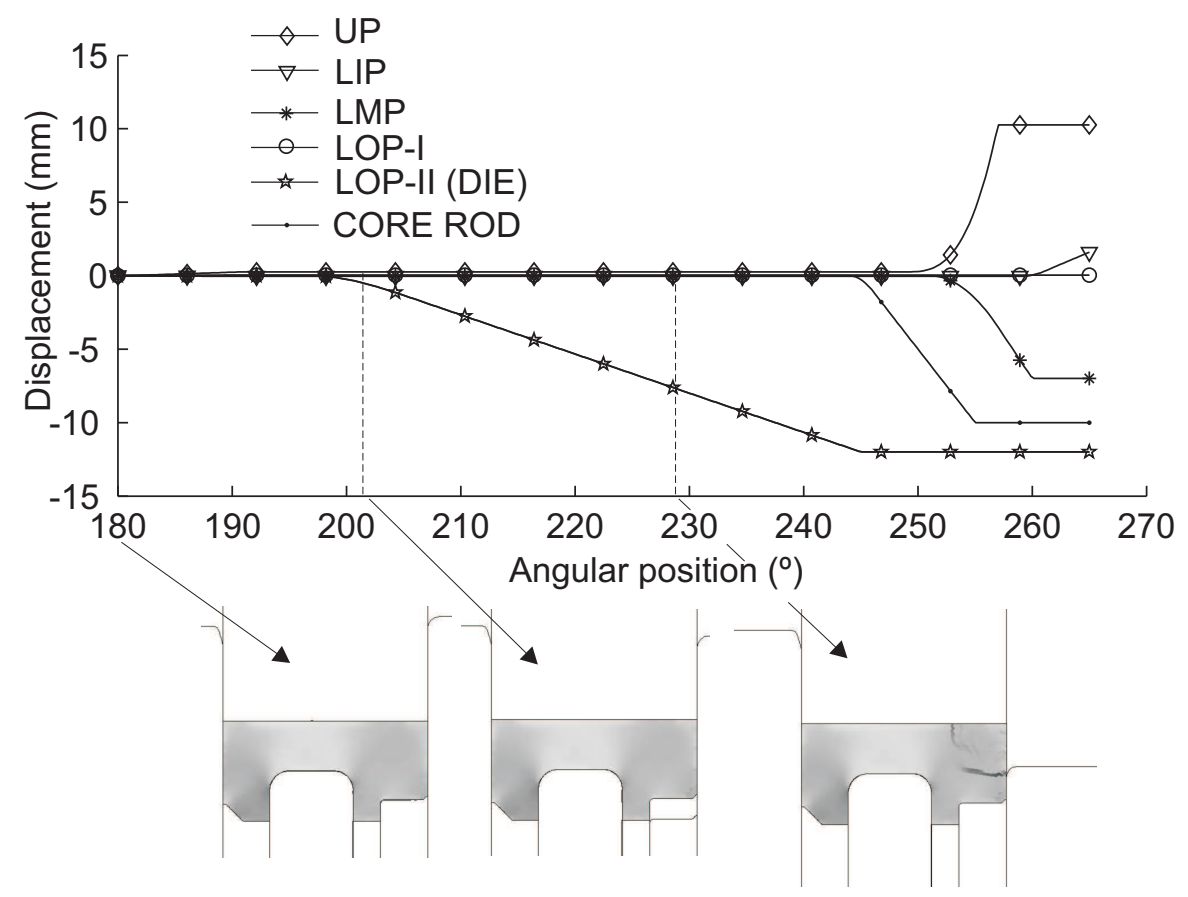

Figure 12: Prescribed punch displacements as a function of the angular position, together with a sequence of contour plots of computed cohesion. Case in which the LMP and the core rod are held stationary.

is, when the main body of the part is clear of the die. The radial expansion of the main body coupled with the radial force exerted on the portion of outer surface still restrained by the die tends to shear the compact along the radial direction, as pictorially depicted in figure 13.b. Accordingly, this presumably shear-mode (or mode-II) crack can be eliminated by reducing the level of radial stress when withdrawing the die. One possibility to carry out this reduction is, as recommended by [15], to decrease the degree of confinement of the part by withdrawing the lower middle punch and the core rod. Another possibility to reduce the effects of radial expansion would be to diminish the hold down pressure exerted by the upper punch. However, this practice may conflict with the requirements for avoiding the crack promoting effect of uncontrolled punch deflections.

The other hypothesis advocates that the root cause of the numerically predicted crack is the "stepped" character of the die. As already mentioned, the lower outer punch LOPII is mounted on the die platen. Thus, as the die descends to free the part, the LOP- 


\section{Cohesion (Mpa)}

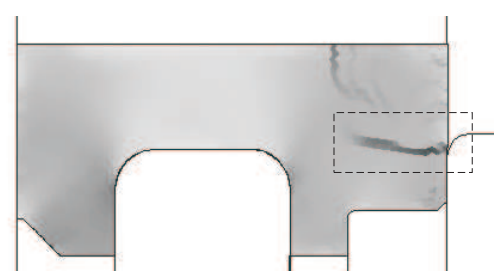

(a)

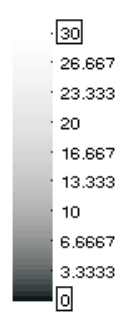

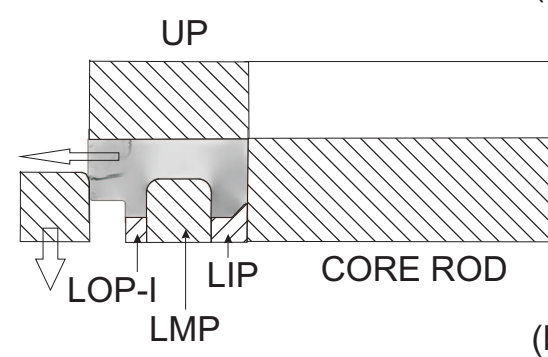

(b)

Expansion

due to

elastic strain

release

Figure 13: (a) Contour plot of computed cohesion at $\phi=203^{\circ}$ for the case in which the LMP and the core rod are held stationary. (b) Schematic representation of the effect of elastic strain release.

II inevitably moves down away from the compact. This separation leaves the external portion of the compact vertically unsupported and, consequently, frictional downward forces between the die and the compact may tend to pull apart the main body and the bottom section. According to this hypothesis, thus, the horizontal crack develops predominantly under opening or mode-I loading conditions. In turn, the bending deformation caused by these frictional forces would also explain consistently the formation of the other discernable de-cohesion path (see figure 13.a), located at the top face of the part.

In order to ascertain which hypothesis is more consistent, we shall carry out two additional finite element analysis of the die withdrawal stage. In the first one, the lower middle punch and the core rod accompany the die in its downward motion so as to release some elastic radial strain. In the second one, the lower inner punch is kept fixed so that it can support the part while the die descends.

\section{Option b: Withdrawing the core rod and the LMP simultaneously}

The tooling displacement diagram, together with a sequence of three contour plots of 


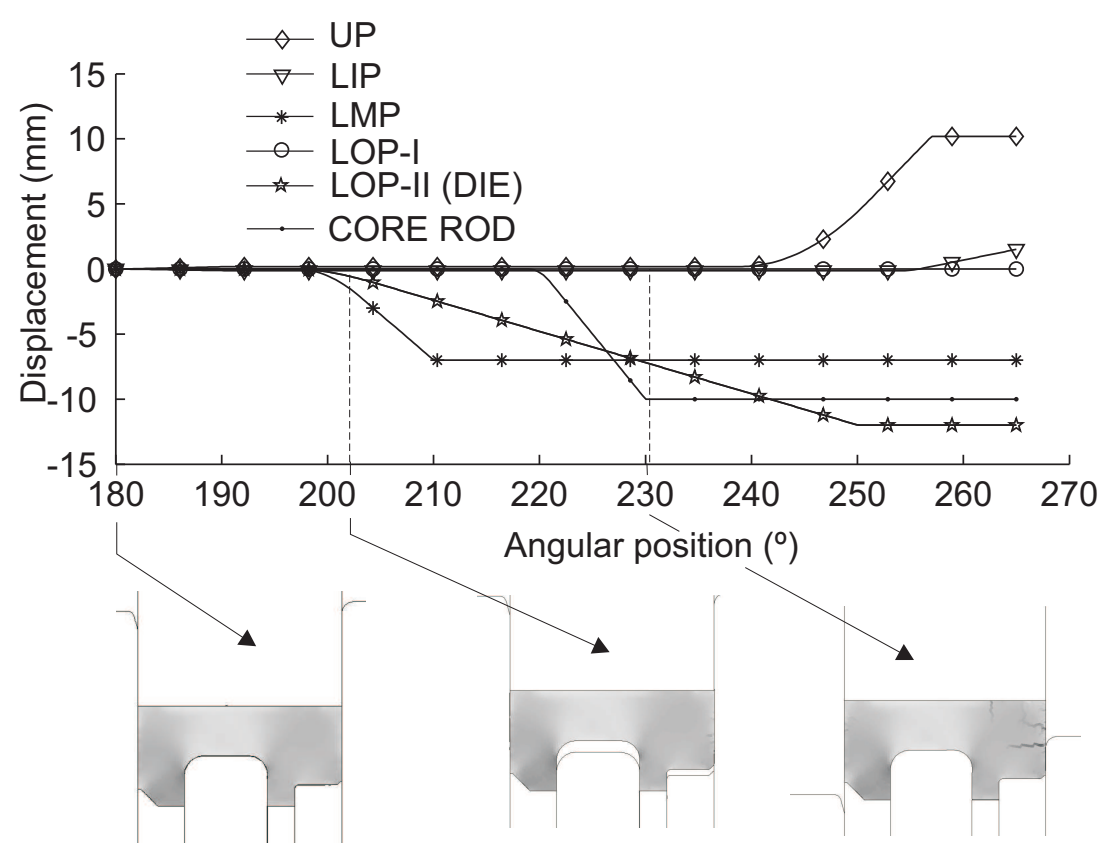

Figure 14: Prescribed punch displacements as a function of the angular position, together with a sequence of contour plots of computed cohesion. Case in which the LMP and the core rod are withdrawn simultaneously with the die.

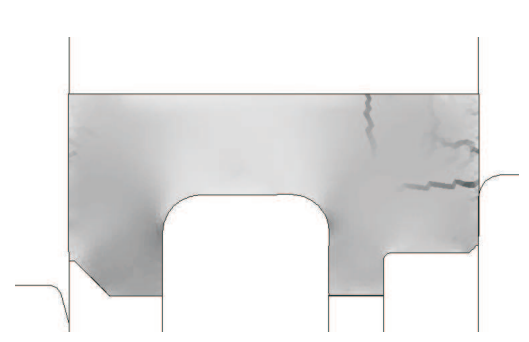

(a)

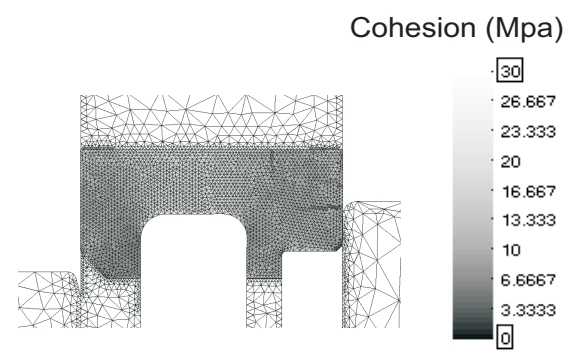

(b)

Figure 15: (a) Contour plot of computed cohesion at $\phi=230^{\circ}$. Case in which the LMP and the core rod are withdrawn simultaneously with the die. for the kinematics shown in figure 12 , for $\phi=230^{\circ}$. (b) The same contour plot showing the mesh used in the computations.

cohesion, corresponding to this case are shown in figure 14. For ease of visualization, an enlarged view of the contour plot at $\varphi=230^{\circ}$ is displayed in figure 15.a. Details of crack propagation through the mesh employed in the calculations can be appreciated in figure 15.b. A qualitative comparison of the contour plot in figure 15.a with that contained in 
figure 13 leads immediately to the conclusion that withdrawing the lower middle punch and the core rod has not proved effective in eliminating the observed cracks. Both contour plots exhibit the same de-cohesion patterns, being the only discernable effect a slight decrease in the intensity of the degradation along these paths.

\section{Option c: Holding stationary the LOP-II.}

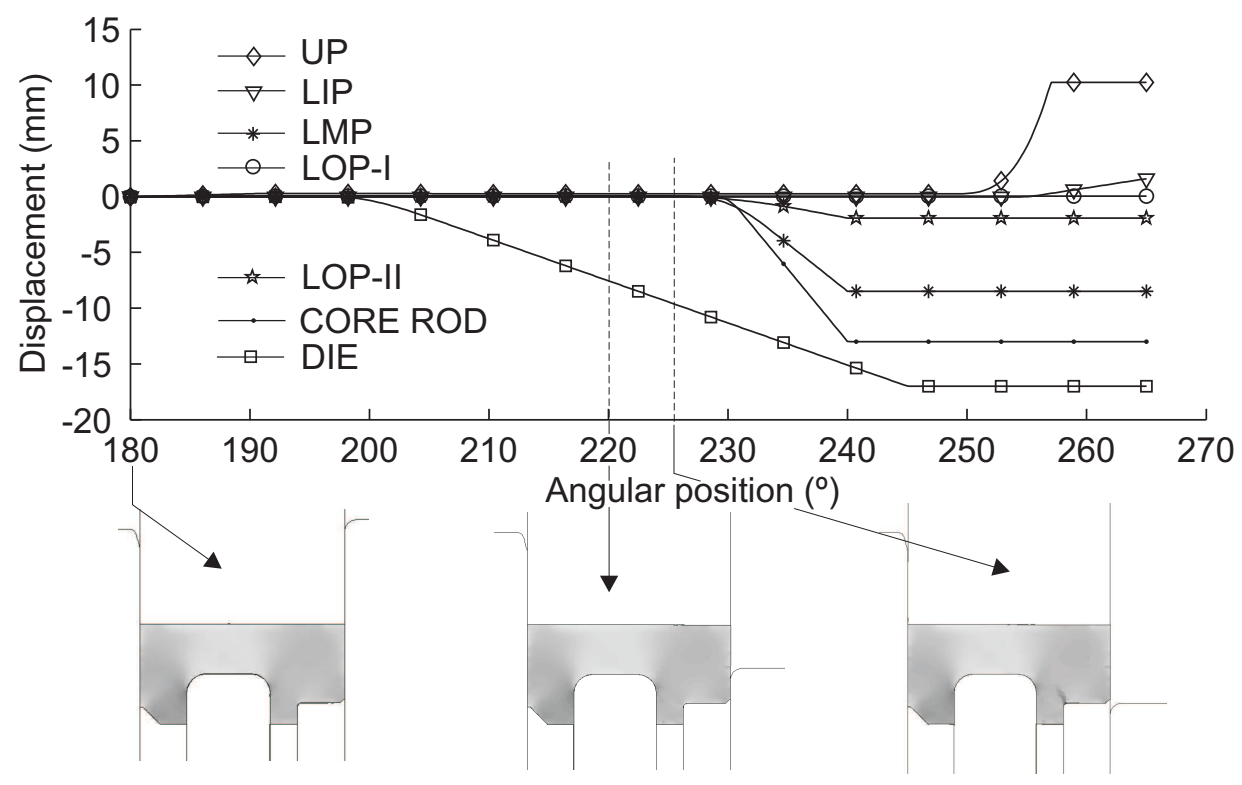

Figure 16: Prescribed punch displacements as a function of the angular position, together with a sequence of contour plots of computed cohesion. Case in which the LOP-II moves independently from the die.

The modeling of this scenario requires to consider a tooling arrangement different from the one described in section 2.2. Rather than attached to the die platen, the LOP-II is assumed to be mounted on an independent platen so that it can support the compact during the downward motion of the die platen. Consequently, in this ejection schedule the part is fully supported by the four lower punches, as indicated in the diagram of tooling displacements shown in figure 16. Below this diagram we show the cohesion distribution at three different times during the die withdrawal. The central plot depicts a situation in which the top face of the die is practically flush with the working end of the LMP, which is the relative position of die and LMP at which the de-cohesion patterns reported in the preceding simulations were detected. In this case, by contrast, the cohesion distribution 


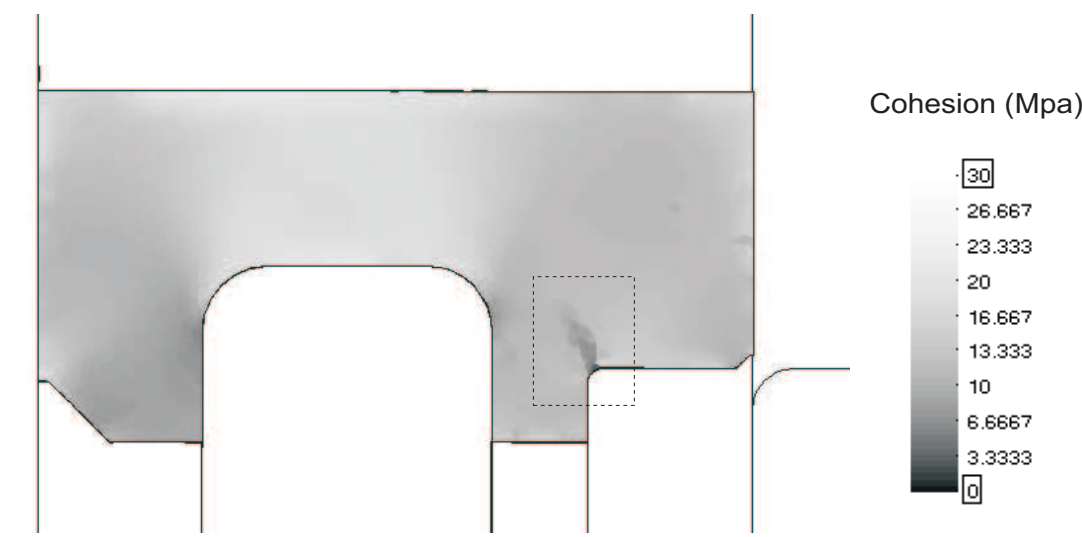

(a)

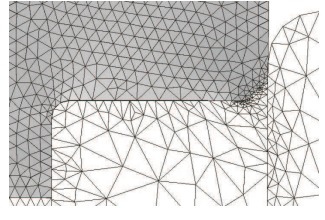

(b)

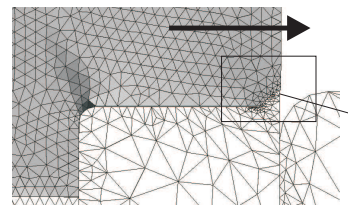

(c)

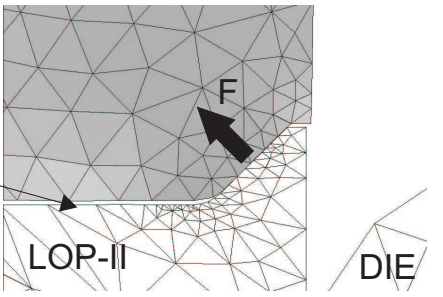

(d)

Figure 17: (a) Contour plot of computed cohesion at $\varphi=226^{\circ}$. Case in which the LOP-II moves independently from the die. (b) (c) Enlarged views of the zone at which the crack is formed (d) Schematic representation of the force generated on the protruding rim due to radial expansion.

exhibits a relatively uniform aspect and no evidence of intense loss of cohesion is observed. This fact clearly substantiates the second hypothesis advanced previously. Thus, we can conclude from the numerical simulations carried out that the root cause of the numerically predicted cracks (shown in figure 13.a) seems to lie in the "stepped" character of the die.

Incidentally, examination of the rightmost contour plot in figure 16 (displayed in magnified form in figure 17.a) shows a vertical, slightly leaned inwards, de-cohesion path that develops at the junction of the levels formed by the LOP-I and the LOP-II. Although the primary goal in studying this ejection schedule was to merely confront the hypothesis advanced at the onset of the discussion, rather than rigorously investigate the defects formed in producing the part using this tooling arrangement ${ }^{3}$, it may prove instructive to, at least superficially, inquire about the root cause of this de-cohesion pattern.

\footnotetext{
${ }^{3}$ In practice, this tooling arrangement with an independently movable LOP-II was not tested due to equipment limitations.
} 
A plausible explanation for this localized loss of cohesion may lie in the particular geometry of the LOP-II. Inspection of the plots in figures 17.b and 17.c indicates that loss of cohesion in the corner region occurs right after the compact is totally freed from the radial restraint imposed by the die. The top surface of the LOP-II is not completely horizontal, but it has a vertical protruding feature $(0.2 \mathrm{~mm}$ height, see figure 1$)$ at the outer edge. As the compact emerges from the die, it tends to expand radially due to elastic strain release. The radial expansion of the lower portion of the part, however, is hindered to some extent by the protruding feature. This restriction generates a lifting force (see figure 17.d), that, considering that the leg section is restrained by the radial action of the LOP-II and the LMP, induces bending of the part. The computed de-cohesion path might be ascribed thus to the effects of such bending deformation.

\section{Ejection of the modified part}

The main implication of the geometry modification introduced in the revised design the abruptness of the cross-sectional change between the outermost levels is eliminated by a tapered surface, see figure 2 - is that it dispenses with the need for two separate lower outer punches. Accordingly, the two thin-walled lower outer punches LOP-I and LOP-II employed in shaping the part in its original conception are replaced by a single, more massive, lower outer punch, abbreviated LOP, with a tapered top surface. Note that this modification is somehow consistent with the hypothesis put forward in the previous section for explaining the difficulties in producing a free-defect part using the "stepped die" tooling configuration.

We bypass the details of the pressing kinematics and started conditions employed to press the part in its revised form, and, in what follows, we concentrate exclusively on presenting the computed results corresponding to the ejection stage; the reader is referred to [16] for further information on pressing conditions with the revised geometry.

The tooling motions corresponding to the ejection of the modified part - which are, incidentally, the motions with which the part was finally manufactured - are displayed in figure 18. In the pressure release stage, the lower outer punch and the lower middle punch are programmed to move upward $0.08 \mathrm{~mm}$ and $0.06 \mathrm{~mm}$, respectively, while maintaining a 
hold down force of 10 Tons. Then, the die is progressively lowered so as to free the part. The downward motion of the die is followed by the withdrawal of the core rod and the lower middle punch. Finally, the upper punch moves up away from the compact and the lower inner punch is slightly lifted so as to ready the part for being removed by the gripper. The cohesion distribution computed at four different positions are shown also in figure 18. It is apparent that the cohesion field remains unaffected during the ejection process. The model predictions, therefore, are in accordance with experimental evidence and confirm the benefits of manufacturing the part according to the revised design.

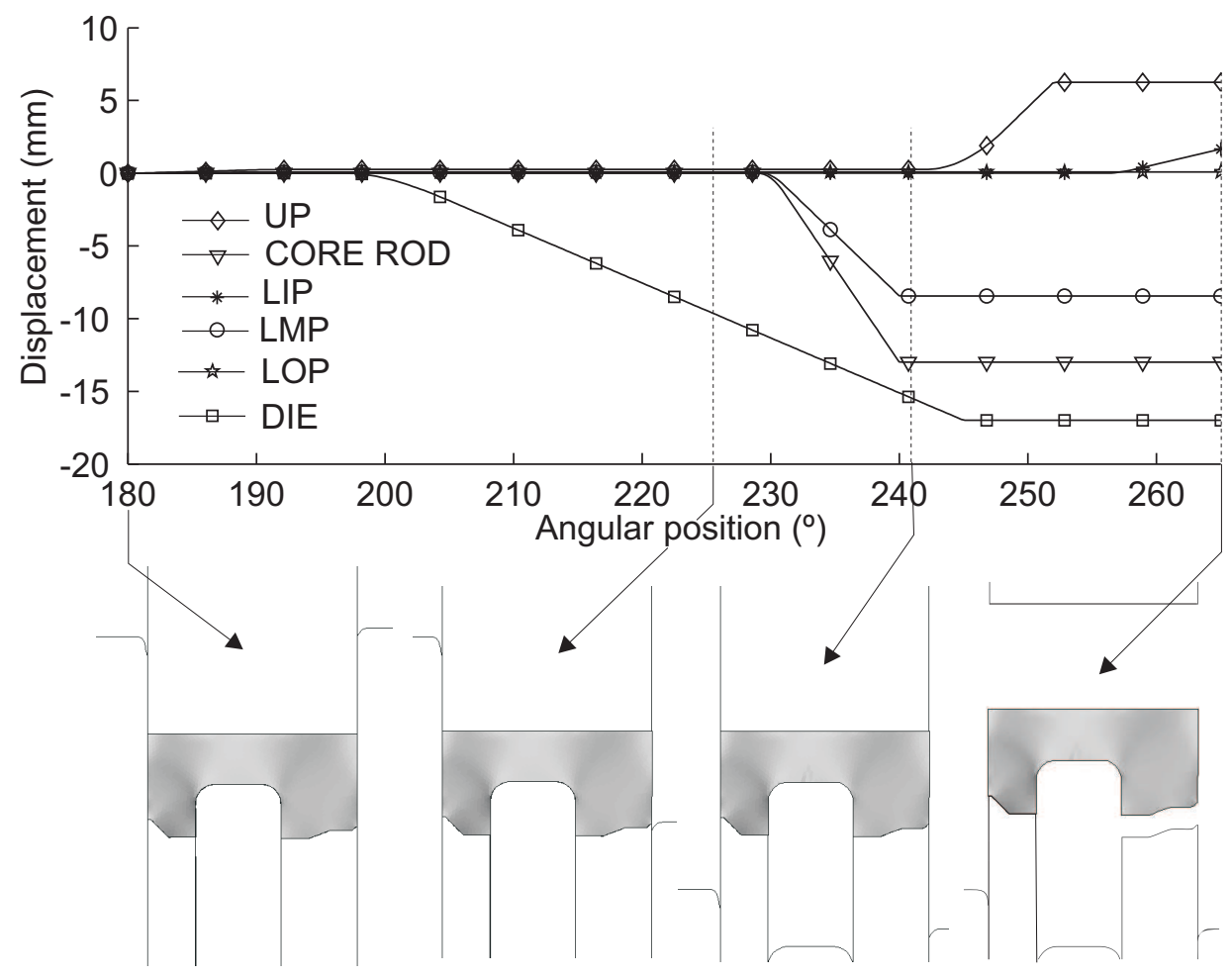

Figure 18: Prescribed punch displacements as a function of the angular position, together with a sequence of contour plots of computed cohesion (modified part).

\subsubsection{Pressure release stage with total axial unloading}

Finally, by way of evaluation of the ability of the model in reproducing other typologies of ejection cracks, we shall numerically examine how the structural integrity of the part would have been affected by a sudden release of the axial force exerted by the upper punch, i.e., 
a case in which the upper punch moves up away from the compact while the lower platens remain fixed (no hold-down force). In figure 19, the graphs of the computed displacement of punch working ends versus the angular position are displayed. Below this diagram, we show a sequence of contour plots of computed cohesion. Examination of these contour plots clearly reveals a horizontal de-cohesion path emanating from the junction corner and propagating outwards through the section change. At the end of the pressure release stage (see figure 20), the computed de-cohesion path spans the whole section, a fact that suggests that fracture may be imminent.

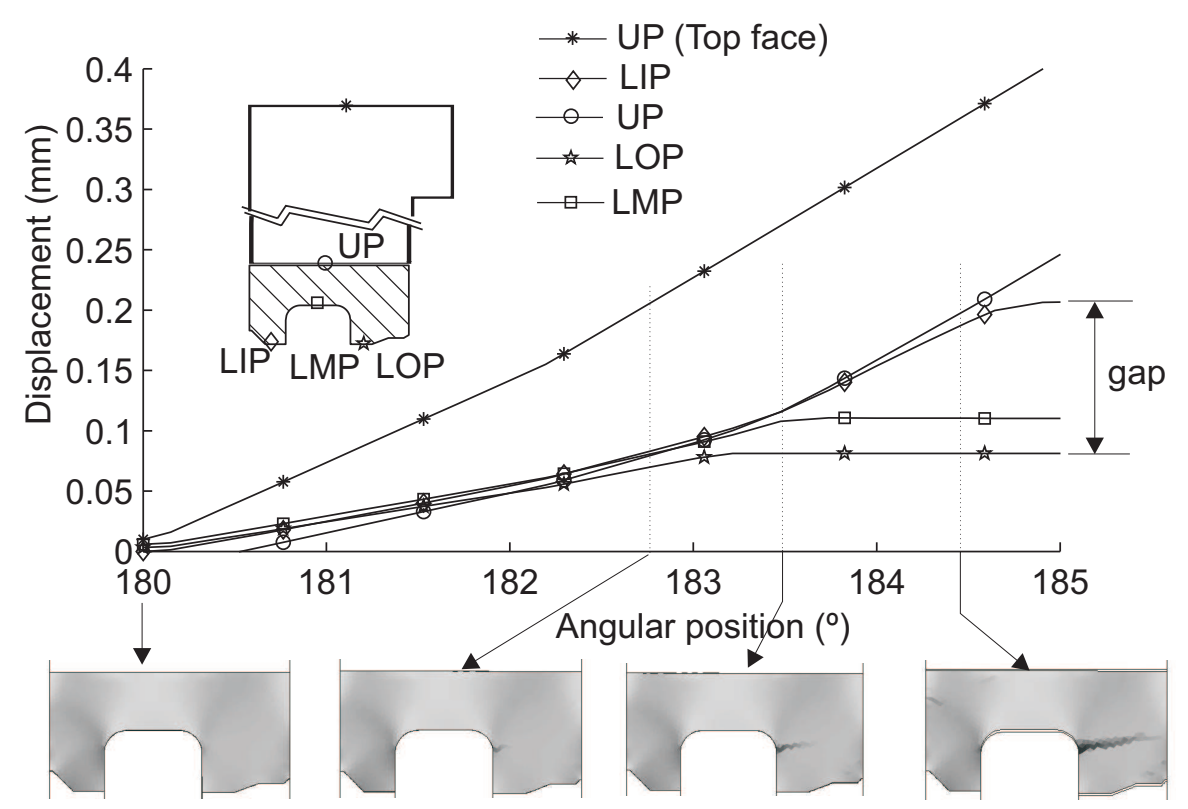

Figure 19: Total axial unloading case (modified part). (a) Displacement of the working ends of lower and upper punches, together with the displacement of the top face of the upper punch, as a function of the angular position. (b) Sequence of contour plots of cohesion.

The origin of this localized loss of cohesion can be readily explained from the punch displacement diagram displayed in figure 19. Since the lower inner punch LIP is larger and has smaller cross-sectional area than the other lower punches, it experiences a larger elastic deflection and, consequently, it pushes the compact upward, generating gaps between the working ends of the LMP and LOP. In this process, the outer flange is partially gripped 


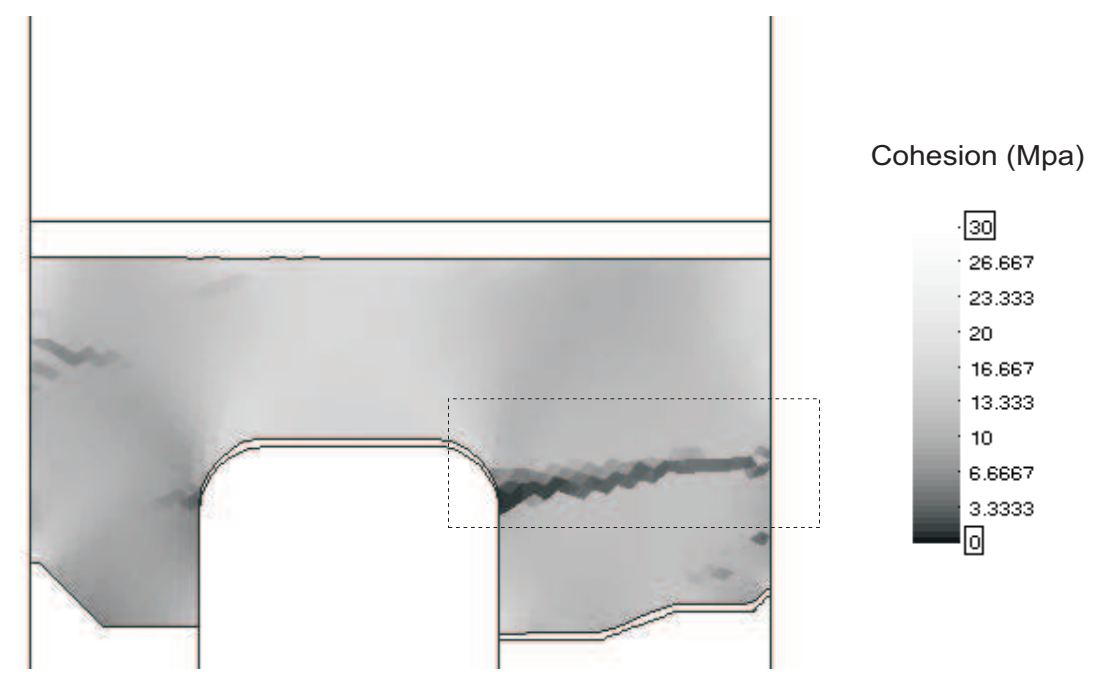

Figure 20: Contour plot of cohesion at the end of pressure release stage. Total axial unloading case (modified part)

between the LMP outer wall and the LOP inner wall, due to friction effects. The combination of this restraining action and the upward movement of the LIP places in tension the corner region and the crack is therefore induced.

\section{Conclusions}

The primary goal of this work was to explore and evaluate the possibilities and limitations of using a finite element model proposed by the authors elsewhere [10] as a tool for assisting in the solution of problems linked with cracks generated during the ejection phase in $\mathrm{P} / \mathrm{M}$ die compaction processes. For this purpose, a carefully detailed case study of the compaction of an axially symmetric, multilevel adapter in an advanced CNC press machine has been performed; mathematical and numerical formalities have been left aside, and attention has been confined exclusively on how information generated from simulations can be "harnessed" to solve a practical problem. Specifically, the aim was to ascertain the root cause of the seemingly inexplicable difficulties encountered by the manufactured in fabricating the concerned part according to initial customer's specifications. Scrutiny and comparison of 


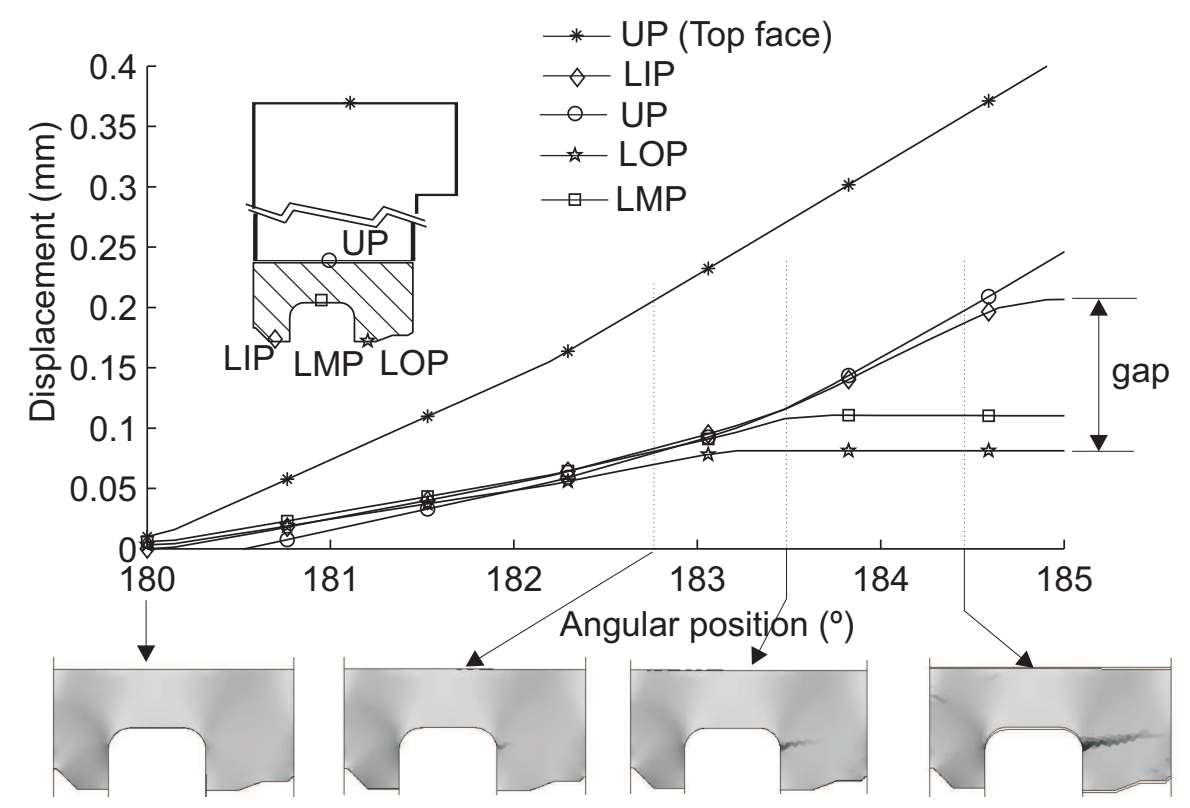

Figure 21: Total axial unloading case (modified part). (a) Displacement of the working ends of lower and upper punches, together with the displacement of the top face of the upper punch, as a function of the angular position. (b) Sequence of contour plots of cohesion.

computed cohesion distributions resulting from alternative ejection routes have allowed us to advance a plausible reason for these difficulties: it seems that the root cause lies in the "stepped" character of the die. The outermost lower punch is mounted on the die table and, thereby, as the die descends, the lower outer punch LOP-II leaves inevitably unsupported the outer portion of the flange; frictional downward forces between the die and the compact tend thus to pull apart the main body and the bottom section.

The final decision of revising and simplifying the initial customer's specifications was motivated by equipment limitations: the employed CNC press only afforded configurations with up to three lower-punch platens. Nevertheless, simulations of an ejection process using a four-lower platen arrangement (see figure 16) have revealed that a likely source of problems in this case might arise from the existence of a protruding rim in the outermost lower punch (LOP-II). According to numerical results, thus, it seems in retrospective that manufacturing the part following the revised design was the right decision. 
The detection of this protruding feature as a potential source of problems illustrates somehow how numerical simulations could be of great assistance also in a prospective examination of the viability of a given tooling arrangement. In this case, model predictions would have suggested that a four-lower platen configuration is also apt to lead to defective parts. Needless to say, whether to use one configuration or other must remain an engineering decision: the role of numerical simulations in the decision-making process should be to guide engineering judgment, not to supplant it.

Indeed, even if every precaution to ensure "correctness" and eliminate numerical errors is taken, the model keeps being but a simplified picture of physical reality; it contains simplifying assumptions, and uncertainty plagues the calibration of material parameters and determination of boundary conditions. It is important to be aware of the predictive limitations arising from this fact, guard against unrealistically high expectations, and know which questions can be conceivably answered and which cannot. For instance, in a prospective study, the numerical simulation of a given ejection route attempts, in principle, to answer the question: will this ejection route lead to a free-defect part ? Such a question, however, demands a categorical yes or no answer: there is no flexibility and the risk of arriving at wrong conclusions is thus high. Except in some pathological cases, such as the uncontrolled punch deflections (see figure 20), one should refrain from drawing definite conclusions from a single simulation. As shown in this work, a more fruitful strategy consists in running several analyses for different alternative ejection procedures, and limiting oneself to answer the question: which of these ejection procedures represents more favorable conditions for ejecting the part safely from the die? Although the answer to this question remains surely uncertain, judicious interpretation and simple qualitative comparisons of cohesion contours may give a hint of which ejection option has the highest probability of success. To handle the inescapable uncertainty associated with modeling errors in a more systematic and rigorous manner, one should abandon the confines of deterministic simulation and move to more sophisticated, statistical-based methods. The work by [12], succinctly outlined in the preamble, provides an excellent guideline for accomplishing this task in the context of $\mathrm{P} / \mathrm{M}$ compaction modeling. 


\section{Acknowledgments}

The Spanish Ministry of Science and Innovation, and the Catalan Government Research Department, are gratefully acknowledged for financial support under grants BIA2008-00411 and 2009 SGR 1510, respectively. The authors wish to thank AMES S.A. for supporting the experimental part of this research; a special debt of gratitude is owed to Enric Snchez and Josep Lluis Celeiro for their endless patience in helping the authors understand the intricacies of CNC press machines.

\section{References}

[1] P. Brewin and L. Federzoni, Powder Metallurgy, 2006, 49, 8-10.

[2] P. Brewin, O. Coube, P. Doremus and J. Tweed: 'Modelling of Powder Die Compaction', Springer Verlag, 2007.

[3] L. Federzoni, H. Riedel, O. Coube, M. Oldenburg, D. Gethin, P. Mosbah, J. Virta, H. Martikainen, A. Frachon, P. Doremus et al., Powder Metall, 1999, 42(4), 301-311.

[4] O. Coube and H. Riedel, Powder Metallurgy, 2000, 43, 123-131.

[5] S. M. Tahir and A. K. Ariffin, International Journal of Solids and Structures, 2006, 43, 1528-1542.

[6] K. Mori, Y. Sato, M. Shiomi and K. Osakada, International Journal of Machine Tools and Manufacture, 1999, 39(7), 1031-1045.

[7] P. Redanz, International Journal of Solids and Structures, 2001, 38(5), 759-775.

[8] P. Jonsén, H.-A. Häggblad and K. Sommer, Powder Technology, 2007, 176, 148-155.

[9] P. Jonsén and H. Häggblad, International Journal of Solids and Structures, 2007, 44, 6398-6411.

[10] J. A. Hernández, J. Oliver, J. C. Cante and R. Weyler, International Journal of Solids and Structures, 2011, 48, 292-316.

[11] J. Hernández, J. Oliver, J. Cante and R. Weyler, International Journal for Numerical Methods in Engineering, 2011.

[12] S. Rolland, D. Gethin, R. Lewis and J. Tweed, Proceedings of the Institution of Mechanical Engineers, Part E: Journal of Process Mechanical Engineering, 2010, 224(3), 203-212.

[13] E. Pavier: 'Caracterisation du comportement d'une poudre de fer pour le procede de compression en matrice', Ph.D. thesis, L'Institut National Polytechnique de Grenoble, 1998.

[14] B. Ferguson and T. Krauss: 'Proceedings of the 1991 Powder Metallurgy Conference and Exhibition', London, 147-164. 
[15] D. Zenger and H. Cai: 'Handbook of the Common Cracks in Green P/M Compacts', Metal Powder Industry Federation, Worcester, 1997.

[16] J. A. Hernández, J. Cante, J. Oliver and R. Weyler, Journal of Materials Processing Technology, 2011, submitted for publication. 